\title{
RAPD Molecular Marker Based Genetic Diversity among Oriental Pickling Melon (Cucumis melo var. conomon) Genotypes in Karnataka, India
}

\author{
L. Mukunda Lakshmi ${ }^{1}$, H.B. Lingaiah ${ }^{1}$, A. Mohan Rao $^{2}$ and A. Ramesh ${ }^{2}$ \\ ${ }^{1}$ Department of Vegetable Science, University of Agricultural Science, GKVK, \\ Bangalore, Karnataka, India \\ ${ }^{2}$ Department of Genetics and Plant Breeding, University of Agricultural Science, \\ GKVK, Bangalore, Karnataka, India \\ *Corresponding author
}

\begin{tabular}{|c|c|}
\hline & A B S T R A C T \\
\hline $\begin{array}{l}\text { Oriental pickling } \\
\text { melon, Cucumis } \\
\text { melo var. conomon. } \\
\text { RAPD, Genetic } \\
\text { diversity }\end{array}$ & \multirow{3}{*}{$\begin{array}{l}\text { Oriental pickling melon (Cucumis melo var. conomon) is one of the melon group } \\
\text { vegetables of the Cucurbitaceae, with high potential as commercial short duration } \\
\text { vegetable in South India. Twenty five RAPD primers were used which generated a total of } \\
182 \text { bands, out of which } 107 \text { bands were polymorphic }(58 \%) \text { revealing a substantial } \\
\text { amount of genetic diversity within this species. Similarity values among all the accessions } \\
\text { ranged from } 0.948 \text { to } 0.619 \text {. According to the dendrogram formed, all the } 15 \text { pickling } \\
\text { melon accessions were divided into three major clusters and four solitary clusters. Cluster } \\
1 \text { consisted of } 2 \text { genotypes, cluster } 2 \text { had four genotypes and cluster } 3 \text { had five genotypes. } \\
\text { The genotype CMC GKVK12 appeared to be most diverse and genetically most similar } \\
\text { genotypes were CMC GKVK14 and } 15 \text {. The RAPD profile identified CMC GKVK12, } \\
\text { CMC GKVK4, CMC GKVK11, and CMC GKVK13 genotypes as the most promising and } \\
\text { also established their inter- relationship, which could be of great significance to choose } \\
\text { them as parents in the future breeding programme. }\end{array}$} \\
\hline Article Info & \\
\hline $\begin{array}{l}\text { Accepted: } \\
10 \text { February } 2017 \\
\text { Available Online: } \\
10 \text { March } 2017\end{array}$ & \\
\hline
\end{tabular}

\section{Introduction}

Oriental pickling melon (Cucumis melo var. conomon) is one among the melon group vegetables belongs to Cucurbitaceae family with a chromosomal number $2 n=24$. It is also called as golden melon or culinary melon in English. Fruits are varying in size, small to medium and big fruits with smooth tender skin, white flesh usually with little sweetness and odour. The fruits which contain moderate amount of vitamins and minerals are used in the preparations of an array of traditional vegetarian dishes like chutney, curry, sambar and pickles. The fruits possess cooling properties and are used as a skin moisturizer and as a digestive agent (George, 2008).

In Karnataka, the crop is cultivated under limited area using mostly local varieties which are low in productivity. There is a need for collection of local genotypes and evaluate them for their adoptability to particular locations especially to their nontraditional areas. A wide range of genetic diversity exists in Karnataka with respect to fruit morphology such as size, shape, fruit flesh thickness and exocarp colour. Molecular markers provide a 
quick and reliable method for estimating genetic diversity among genotypes (Thormann et al., 1994). In order to develop a reliable tool, efforts were therefore made to develop DNA finger print profile of cultivars to establish a relationship and also to understand the genetic distance which can be used for future breeding programme. The DNA finger print profile would supplement the existing DUS characterization till the molecular characterization is widely adopted.

\section{Materials and Methods}

Fifteen morphologically and geographically distinct pickling melon accessions (Table 1) were collected from different parts of South Karnataka and then grown in Summer, 2009 in the experimental plots of Vegetable Seed Production Unit (VSPU), Department of Horticulture, University of Agricultural Sciences (UAS), Gandhi Krishi Vignana Kendra (GKVK), Bangalore. The genomic DNA was isolated from green and healthy leaves of twenty days old seedlings by cetyltrimethyl ammonium bromide (CTAB) method (Doyle and Doyle, 1990) with minor modifications. The extracted stock DNA was quantified on 0.8 per cent agarose using standard lambda uncut DNA and the DNA samples were diluted to $25 \mathrm{ng} / \mu \mathrm{l}$ prior to polymerase chain reaction (PCR) amplification.

PCR DNA amplification was performed by using fifteen Operon decamer primers with 50-70 per cent GC content (Operon Technologies, USA) and ten Sigma decamer primers (Sigma Aldrich, USA). The polymerase chain reactions was performed in a $10 \mu 1$ mixture containing $25 \eta \mathrm{g} / \mu 1 \mathrm{DNA}, 10$ $\mu \mathrm{M} / \mu \mathrm{l}$ Primer, 10X Taq assay Buffer, $1 \mathrm{mM}$ dNTPs, $1 \mathrm{U}$ Taq Polymerase and $25 \mathrm{mM}$ $\mathrm{MgCl}_{2}$. Amplifications were performed in a thermal cycler (MJ Research Inc., USA). The standardized PCR include: initial denaturation at $94^{\circ} \mathrm{C}$ for 3 minutes followed by 35 cycles of denaturation at $94^{\circ} \mathrm{C}$ for 30 seconds; primer annealing $38^{\circ} \mathrm{C}$ for 1 minute; primer extension at $72^{\circ} \mathrm{C}$ for 1 minute and a final primer extension at $72^{\circ} \mathrm{C}$ for 10 minutes. The amplicons were assessed by loading on to 1.5\% Agarose along with bromophenol blue as loading dye. Amplicons were separated in an electrophoresis unit at $100 \mathrm{~V}$ for three hours using $1 \mathrm{X}$ TBE buffer and stained with ethidium bromide $(50 \mathrm{mg} / 100 \mathrm{ml}$ agarose) (PALTE 1). The amplification products were scored across the lanes. The score ' 1 ' was assigned for the presence of band and ' 0 ' for absence of band at each loci. The variation in band intensity is not taken into consideration to avoid confusion in scoring. The binary data was used to estimate pair wise genetic distance based on Jaccard's similarity coefficients and a dendrogram was generated based on the unweighed pair group method for arithmatic mean (UPGMA) using software NTSYS-pc version 2.0.

\section{Results and Discussion}

In the RAPD marker analysis, a total of 182 bands were generated in fifteen accessions of pickling melon based on 25 RAPD primers. Of these 107 bands were polymorphic (58\%). On an average 25 primers could amplify 58.79 per cent polymorphic loci (Table 2). Polymorphism within the pickling melon germplasm was found to be high, which reveals a substantial amount of genetic diversity within the species.

The range of amplified bands was between 4 and 12 with an average number of 7.28 bands per primer. OPD- 09 primer gave maximum number (12) of amplified bands, while lowest number (4) was generated by OPA-01, OPB10 and OPC-05 primers. Average number of polymorphic bands per primer was 4.28, while highest polymorphism $(100 \%)$ was revealed by the OPA-01, OPA-11, OPA-12 
and OPD-05 primers. Contrasting to these, Primer-20 exhibited no polymorphism at all. The number of polymorphic loci per primer in the present study is comparable to that reported by Feyzian et al., (2007) in Iranian melon and by Garcia et al., (1998) in melon.

Genetic distance was measured using Jaccard's similarity coefficient and the values ranged from 0.948 to 0.619 (Table 3). Maximum similarity (0.948) was recorded between the genotypes CMC GKVK14 and CMC GKVK15. Both the genotypes were collected from same localities. However, minimum genetic similarity was found between the genotypes CMC GKVK10 and CMC GKVK13 (0.619). Luan et al., (2008) have reported an average dissimilarity of 0.53 \pm 0.14 between any two pairs of accessions based on RAPD assay in Chinese melon, while (Matsui et al., 2002) reported an average dissimilarity of 0.240 in oriental pickling melon.

In the UPGMA dendrogram (Fig. 1) all the 15 pickling melon accessions divided into three major clusters and four solitary clusters. Genotypes such as CMC GKVK4, CMC GKVK11, CMC GKVK12 and CMC GKVK13 fell into solitary clusters.

Table.1 Pickling melon working collections

\begin{tabular}{|l|l|l|l|}
\hline S.No. & $\begin{array}{l}\text { Accession } \\
\text { Number }\end{array}$ & Source & Fruit characters \\
\hline 1. & CMC GKVK-1 & UAS, GKVK, Bangalore & Fruit rind \\
\hline 2. & CMC GKVK-2 & UAS, GKVK, Bangalore & Greenish yellow streaked \\
\hline 3. & CMC GKVK-3 & UAS, GKVK, Bangalore & Greenish yellow striped \\
\hline 4. & CMC GKVK-4 & UAS, GKVK, Bangalore & Greenish yellow striped \\
\hline 5. & CMC GKVK-5 & Shiriyara & Greenish yellow striped \\
\hline 6. & CMC GKVK-6 & Satkatu & Greenish yellow striped \\
\hline 7. & CMC GKVK-7 & Satkatu & Greenish yellow striped \\
\hline 8. & CMC GKVK-8 & Kollebylu & Greenish brown speckled \\
\hline 9. & CMC GKVK-9 & $\begin{array}{l}\text { Parampalli,Salegrama, Kundapura } \\
\text { Taluk }\end{array}$ & Greenish yellow striped \\
\hline 10. & CMC GKVK-10 & $\begin{array}{l}\text { Udli,Barkuru, Brahmavara, Udupi } \\
\text { (District) }\end{array}$ & Greenish brown speckled \\
\hline 11. & CMC GKVK-11 & $\begin{array}{l}\text { Barkuru, Brahmavara, Udupi } \\
\text { (District) }\end{array}$ & Greenish yellow streaked \\
\hline 12. & CMC GKVK-12 & $\begin{array}{l}\text { Hanehalli, Barkuru, Brahmavara, } \\
\text { Udupi (District) }\end{array}$ & Orange streaked \\
\hline 13. & CMC GKVK-13 & $\begin{array}{l}\text { Hanehalli, Barkuru, Brahmavara, } \\
\text { Udupi (District) }\end{array}$ & $\begin{array}{l}\text { Greenish } \\
\text { speckled }\end{array}$ \\
\hline 14. & CMC GKVK-14 & $\begin{array}{l}\text { Keraviske village, Karkala, Udupi } \\
\text { (District) }\end{array}$ & Greenish yellow streaked \\
\hline 15. & CMC GKVK-15 & $\begin{array}{l}\text { Mannur } \\
\text { Udupi (District) }\end{array}$ & Greenish brown speckled \\
\hline
\end{tabular}


Table.2 Polymorphism in pickling melon genotypes as influenced by RAPD primers

\begin{tabular}{|c|c|c|c|c|c|}
\hline \multirow[b]{2}{*}{ Sl. No } & \multirow{2}{*}{$\begin{array}{l}\text { RAPD } \\
\text { Primer }\end{array}$} & \multirow[t]{2}{*}{ Sequence } & \multicolumn{2}{|c|}{ Number of bands } & \multirow[b]{2}{*}{ Polymorphism \% } \\
\hline & & & Total & Polymorphic & \\
\hline 1 & OPF-08 & GGG ATA TCG G & 9 & 6 & 66.67 \\
\hline 2 & OPD-09 & CTC TGG AGA C & 12 & 8 & 66.67 \\
\hline $3 *$ & OPA-11 & CAA TCG CCG T & 6 & 6 & 100.00 \\
\hline $4 *$ & OPA-12 & TCG GCG ATA G & 5 & 5 & 100.00 \\
\hline 5 & OPC-06 & GAA CGG ACT C & 7 & 6 & 85.71 \\
\hline $6^{*}$ & OPD-05 & TGA GCG GAC A & 6 & 6 & 100.00 \\
\hline $7 *$ & OPA-01 & CAG GCC CTT C & 4 & 4 & 100.00 \\
\hline 8 & OPB-08 & GTC CAC ACG G & 5 & 4 & 80.00 \\
\hline 9 & OPB-10 & CTG CTG TTA C & 4 & 3 & 75.00 \\
\hline 10 & OPE-03 & CCA GAT GCA C & 10 & 8 & 80.00 \\
\hline 11 & OPE-06 & AAG ACC CCT C & 7 & 6 & 85.70 \\
\hline 12 & OPB-04 & GGA CTG CTC C & 8 & 7 & 85.70 \\
\hline 13 & OPE-11 & GAG TCT CAG G & 6 & 5 & 83.33 \\
\hline 14 & OPA-02 & TGC CGA GCT G & 7 & 4 & 57.14 \\
\hline 15 & OPC-05 & GAT GAC CGC C & 4 & 2 & 50.00 \\
\hline 16 & Primer-10 & TAG CCC GCT T & 9 & 7 & 77.78 \\
\hline 17 & Primer-4 & GGG CAA TGA T & 9 & 3 & 33.33 \\
\hline 18 & Primer-25 & GCG GTT GAG G & 11 & 4 & 36.36 \\
\hline 19 & Primer-18 & CCC GTT TTG T & 8 & 5 & 62.50 \\
\hline 20 & Primer-19 & ATT GGG CGA T & 8 & 2 & 25.00 \\
\hline 21 & Primer-20 & GAA TTT CCC C & 8 & 0 & 0 \\
\hline 22 & Primer-33 & GGA AAC CTC T & 9 & 1 & 11.11 \\
\hline 23 & Primer-7 & CTG TCC CTT T & 6 & 2 & 33.33 \\
\hline 24 & Primer-36 & TAC GTC TTG C & 8 & 2 & 25.00 \\
\hline 25 & Primer-5 & TGC GCC CTT C & 6 & 1 & 16.67 \\
\hline \multicolumn{3}{|c|}{ Total } & 182 & 107 & \\
\hline \multicolumn{3}{|c|}{ Average } & 7.28 & 4.28 & 58.79 \\
\hline
\end{tabular}




\section{Int.J.Curr.Microbiol.App.Sci (2017) 6(3): 324-330}

Table.3 Similarity matrix of 15 pickling melon genotypes according to Jaccard's coefficient

\begin{tabular}{|c|c|c|c|c|c|c|c|c|c|c|c|c|c|c|c|}
\hline & $\begin{array}{l}\text { CMC } \\
\text { GKVK1 }\end{array}$ & $\begin{array}{l}\text { CMC } \\
\text { GKVK2 }\end{array}$ & $\begin{array}{l}\text { CMC } \\
\text { GKVK3 } \\
\end{array}$ & $\begin{array}{l}\text { CMC } \\
\text { GKVK4 } \\
\end{array}$ & $\begin{array}{l}\text { CMC } \\
\text { GKVK5 }\end{array}$ & $\begin{array}{l}\text { CMC } \\
\text { GKVK6 } \\
\end{array}$ & $\begin{array}{l}\text { CMC } \\
\text { GKVK7 }\end{array}$ & $\begin{array}{l}\text { CMC } \\
\text { GKVK8 }\end{array}$ & $\begin{array}{l}\text { CMC } \\
\text { GKVK9 }\end{array}$ & $\begin{array}{l}\text { CMC } \\
\text { GKVK10 }\end{array}$ & $\begin{array}{l}\text { CMC } \\
\text { GKVK11 }\end{array}$ & $\begin{array}{l}\text { CMC } \\
\text { GKVK12 }\end{array}$ & $\begin{array}{l}\text { CMC } \\
\text { GKVK13 }\end{array}$ & $\begin{array}{l}\text { CMC } \\
\text { GKVK14 }\end{array}$ & $\begin{array}{l}\text { CMC } \\
\text { GKVK15 }\end{array}$ \\
\hline $\begin{array}{l}\text { CMC } \\
\text { GKVK1 }\end{array}$ & 1.000 & & & & & & & & & & & & & & \\
\hline $\begin{array}{l}\text { CMC } \\
\text { GKVK2 }\end{array}$ & 0.669 & 1.000 & & & & & & & & & & & & & \\
\hline $\begin{array}{l}\text { CMC } \\
\text { GKVK3 }\end{array}$ & 0.696 & 0.925 & 1.000 & & & & & & & & & & & & \\
\hline $\begin{array}{l}\text { CMC } \\
\text { GKVK4 }\end{array}$ & 0.728 & 0.729 & 0.716 & 1.000 & & & & & & & & & & & \\
\hline $\begin{array}{l}\text { CMC } \\
\text { GKVK5 }\end{array}$ & 0.667 & 0.865 & 0.877 & 0.781 & 1.000 & & & & & & & & & & \\
\hline $\begin{array}{l}\text { CMC } \\
\text { GKVK6 }\end{array}$ & 0.761 & 0.758 & 0.752 & 0.804 & 0.772 & 1.000 & & & & & & & & & \\
\hline $\begin{array}{l}\text { CMC } \\
\text { GKVK7 }\end{array}$ & 0.773 & 0.757 & 0.782 & 0.727 & 0.799 & 0.918 & 1.000 & & & & & & & & \\
\hline $\begin{array}{l}\text { CMC } \\
\text { GKVK8 }\end{array}$ & 0.735 & 0.802 & 0.780 & 0.702 & 0.790 & 0.849 & 0.892 & 1.000 & & & & & & & \\
\hline $\begin{array}{l}\text { CMC } \\
\text { GKVK9 }\end{array}$ & 0.718 & 0.786 & 0.796 & 0.769 & 0.767 & 0.785 & 0.802 & 0.885 & 1.000 & & & & & & \\
\hline $\begin{array}{l}\text { CMC } \\
\text { GKV10 }\end{array}$ & 0.774 & 0.662 & 0.657 & 0.742 & 0.658 & 0.808 & 0.783 & 0.770 & 0.736 & 1.000 & & & & & \\
\hline $\begin{array}{l}\text { CMC } \\
\text { GKV11 }\end{array}$ & 0.641 & 0.767 & 0.775 & 0.690 & 0.805 & 0.719 & 0.729 & 0.795 & 0.753 & 0.662 & 1.000 & & & & \\
\hline $\begin{array}{l}\text { CMC } \\
\text { GKV12 }\end{array}$ & 0.729 & 0.652 & 0.654 & 0.653 & 0.674 & 0.707 & 0.698 & 0.719 & 0.682 & 0.737 & 0.627 & 1.000 & & & \\
\hline $\begin{array}{l}\text { CMC } \\
\text { GKV13 } \\
\end{array}$ & 0.632 & 0.777 & 0.737 & 0.640 & 0.769 & 0.683 & 0.724 & 0.765 & 0.710 & 0.619 & 0.748 & 0.667 & 1.000 & & \\
\hline $\begin{array}{l}\text { CMC } \\
\text { GKV14 }\end{array}$ & 0.692 & 0.837 & 0.840 & 0.760 & 0.891 & 0.745 & 0.769 & 0.754 & 0.754 & 0.650 & 0.794 & 0.725 & 0.807 & 1.000 & \\
\hline $\begin{array}{l}\text { CMC } \\
\text { GKV15 }\end{array}$ & 0.652 & 0.830 & 0.848 & 0.722 & 0.871 & 0.732 & 0.750 & 0.741 & 0.745 & 0.642 & 0.812 & 0.683 & 0.828 & 0.948 & 1.000 \\
\hline
\end{tabular}


Fig.1 Dendrogram indicating diversity and relationship among 15 pickling melon genotypes

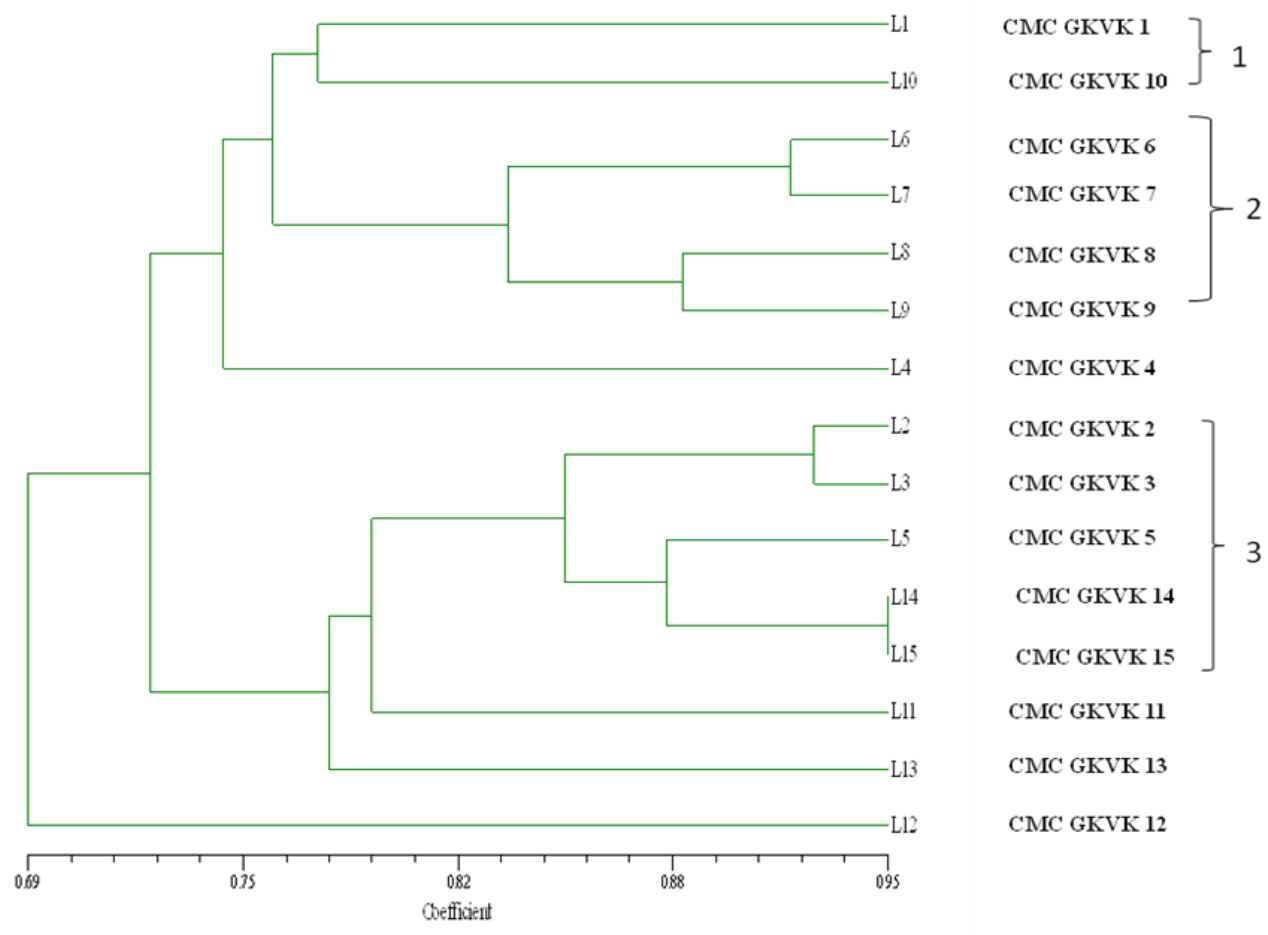

Plate.1 RAPD gel profile of 15 pickling melon accessions amplified by using OPD-5, OPC-6, OPE-11 and OPF-8 primers
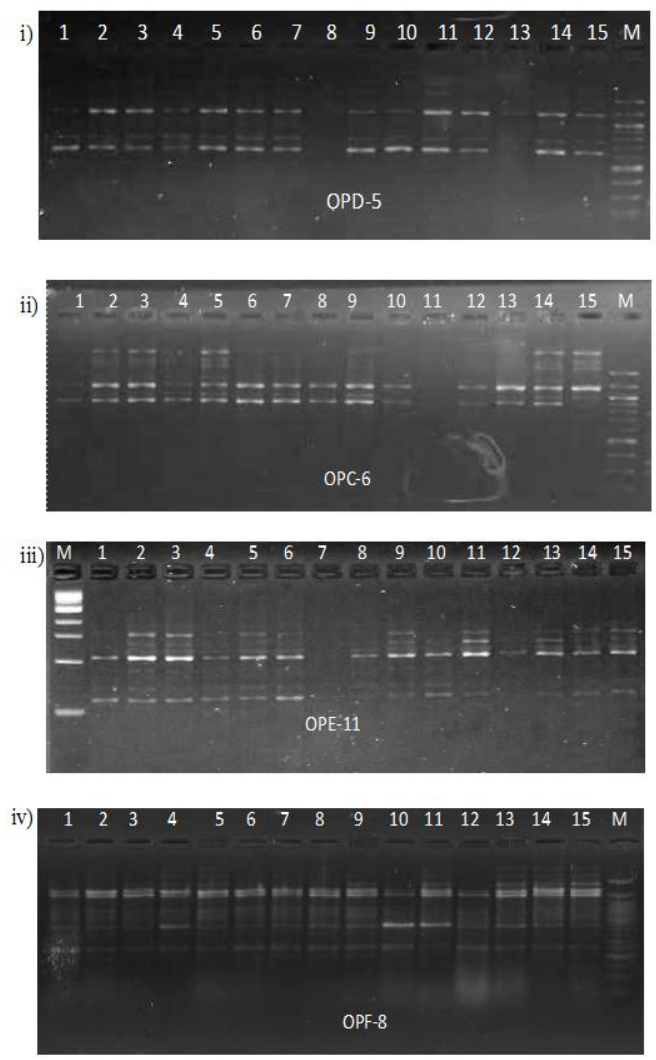
Cluster 1 consisted of 2 genotypes (CMC GKVK1 and CMC GKVK 10), cluster 2 had four genotypes (CMC GKVK6, CMC GKVK7, CMC GKVK8 and CMC GKVK9) and cluster 3 had five genotypes (CMC GKVK2, CMC GKVK3, CMC GKVK5, CMC GKVK14 and CMC GKVK15), which were divided into two sub-clusters (sub cluster I and II). In sub cluster II, CMC GKVK14 and CMC GKVK15 accessions showed the highest similarity. Accession CMC GKVK12 was distinctly separated from all the accessions in the RAPD dendrogram with similarity value of 0.69 and it was the most distinct from other accessions. Previous studies on cluster analysis reported two major clusters among South and East Asian melon types (Tanaka et al., 2007) and five distinct groups among African melon germplasm (Mliki et al., 2001). The most promising accessions based on RAPD analysis were CMC GKVK12, CMC GKVK4, CMC GKVK11, and CMC GKVK13. These accessions need to be further evaluated and could be used either as direct selections or in the crop improvement programme. The information obtained from this study may be useful for identification of promising genotypes and understanding the genetic diversity present in this species.

\section{References}

Doyle, J.J. and Doyle, J.L. 1987. Isolation of plant DNA from fresh tissue. Focus, 12: 13-15.

Feyzian, E., Javaran, J., Deh Ghani, H. and Zamyad, H. 2007. Analysis of the genetic diversity among some of Iranian melon (Cucumis melo L.) land races using morphological and RAPD markers. J. Sci. Technol. Agric. Natur. Resour., 11(41): 163.

Garcia, E., Jamilena, M., Alvarez, J.I., Arnedo, T., Oliver, J.L. and Lozano, R. 1998. Genetic relationships among melon breeding lines revealed by RAPD markers and agronomic traits. Theor. Appl. Genet., 96: 878-885.

George, T.E. 2008. Kanivellari Visual treat, Kerala Call. Monthly, 3: 36-37.

Luan, F., Delannay, I. and Staub, J.E. 2008. Chinese melon (Cucumis melo L.) diversity analyses provide strategies for germplasm curation, genetic improvement and evidentiary support of domestication patterns. Euphytica, 164: 445-461.

Matsui, T., Kosugi, Y., Yanagi, T., Suzuki, H., Pankaj Kumar, B. and Sukarakarn, S. 2002. Classification of oriental melon by RAPD analysis. Pakistan J. Biol. Sci., 5(2): 208-212.

Mliki, A., Staub, J.E., Zhangyong, S. and Ghorbel, A. 2001. Genetic diversity in melon (Cucumis melo L.): An evaluation of African germplasm. Gen. Res. Crop. Evol., 48: 587-597.

Thormann, C.E., Ferreira, M.E., Camargo, L.E.A., Tivang, J.G. and Cosborn, 1994. Comparison of RFLP and RAPD markers to estimation genetic relationship within and among Cruciferous spp. Theor. Appl. Genet., 88: 973-980.

Tanaka, K., Nishithani, A., Akashi, Y., Sakata, Y., Nishida, H., Yoshino, H. and Kato, K. 2007. Molecular characterization of South and East Asian melon, Cucumis melo L. and the origin of group conomon var.makuwa and var. conomon revealed by RAPD analysis. Euphytica, 153: 233247.

\section{How to cite this article:}

Mukunda Lakshmi, L., H.B. Lingaiah, A. Mohan Rao and Ramesh, A. 2017. RAPD Molecular Marker Based Genetic Diversity among Oriental Pickling Melon (Cucumis melo var. conomon) Genotypes in Karnataka, India. Int.J.Curr.Microbiol.App.Sci. 6(3): 324-330.

doi: https://doi.org/10.20546/ijcmas.2017.603.036 